\title{
PERFECT IMAGES OF $p$-SPACES
}

\author{
JÓZEF CHABER
}

\begin{abstract}
We give a new proof of the fact that paracompact $p$-spaces are preserved by perfect mappings. This proof gives more information about the image space than the preceding proofs. We construct some examples showing that the information we get is complete in a way, and an example showing that a perfect image of a $\sigma$-paralindelöf and screenable $p$-space need not be a $p$-space.
\end{abstract}

We shall use the terminology and notation from [E]. All mappings are assumed to be continuous and onto and all spaces are at least regular.

We shall consider the following property of a sequence $\left\{D_{n}\right\}_{n>1}$ of subsets of a space $X$.

(p) If $\bigcap_{n>1} D_{n} \neq \varnothing$, then $\cap\{\bar{A}: A \in \mathbb{Q}\} \neq \varnothing$ for any centered collection $\mathbb{Q}$ of subsets of $X$ containing $\left\{D_{n}: n \geqslant 1\right\}$.

A space $X$ is said to be a $p$-space [A] if $X$ has a sequence $\left\{\mathcal{U}_{n}\right\}_{n>1}$ of open covers such that each sequence $\left\{U_{n}\right\}_{n>1}$ with $U_{n} \in \mathcal{U}_{n}$, for $n \geqslant 1$, has the property (p) [P].

If $X$ is, in addition, a paracompact space, then $X$ has a perfect mapping onto a metric space such that each fiber of this mapping is contained in $\bigcap_{n>1} U_{n}$ for a certain sequence $\left\{U_{n}\right\}_{n>1}$ with $U_{n} \in \mathcal{U}_{n}$ for $n \geqslant 1$.

1. Perfect images of paracompact $p$-spaces. We shall consider the following situation: $g: X \rightarrow S$ is a perfect mapping of $X$ onto a metric space $S$ and $f: X \rightarrow Y$ is a perfect mapping of $X$ onto $Y$. It is known [F, I] that there exists a perfect mapping $h: Y \rightarrow T$ of $Y$ onto a metric space $T$.

From the results of [Ch1, Ch2] it follows that, if $Y$ is a perfect space, then one can find such an $h$ satisfying, for $y \in Y$,

$$
h^{-1} h(y) \subset\left\{y^{\prime}: g f^{-1}\left(y^{\prime}\right)=g f^{-1}(y)\right\} \subset \bigcap\left\{f\left(g^{-1} g(x)\right): x \in f^{-1}(y)\right\} .
$$

In particular, each fiber of $h$ is contained in the image of a fiber of $g$ and consequently, the fibers of $h$ are metrizable (countable or finite) if the fibers of $g$ are metrizable (countable or finite).

In the second section we shall show that this may not be the case if $Y$ is not a perfect space. However, we have

THEOREM 1.1. Assume that $f: X \rightarrow Y$ is a perfect mapping. If $X$ has a perfect mapping $g$ onto a metric space $S$, then $Y$ has a perfect mapping $h$ onto a metric space $T$ such that, for each $y \in Y$, there exists a $y^{\prime} \in Y$ satisfying $h^{-1} h(y) \subset f\left(g^{-1} g\left(f^{-1}\left(y^{\prime}\right)\right)\right)$.

Received by the editors March 12, 1981 and, in revised form, July 28, 1981.

1980 Mathematics Subject Classification. Primary 54C10, 54E18. 
Before proving Theorem 1.1, we list some consequence of this result. We assume that $f, g$ and $h$ are as in the formulation of Theorem 1.1.

COROllary 1.1. Assume that the fibers of $f$ are countable. If the fibers of $g$ are metrizable, then the fibers of $h$ are metrizable too.

Proof. Each fiber of $h$ is a compact subset of the image of a countable union of fibers of $g$ and is therefore metrizable [E, 3.1.20].

COROLlARY 1.2. Assume that the fibers of $f$ are countable ( finite). If the fibers of $g$ are countable (finite), then the fibers of h are countable (finite) too.

COROLlaRY 1.3. Assume that $f$ is at most $k$-to-one. If $g$ is at most $n$-to-one, then the fibers of $h$ are of cardinality not greater than $k(n-1)+1$.

Proof of Theorem 1.1. For $n \geqslant 1$ let $\varepsilon_{n}$ be a locally finite closed cover of $X$ obtained by taking inverse images under the mapping $g$ of elements of a locally finite closed cover of $S$ consisting of sets of the diameter not greater than $\frac{1}{n}$. Clearly, for $x \in X$, the sequence $\left\{\operatorname{St}\left(x, \mathcal{E}_{n}\right)\right\}_{n \geqslant 1}$ satisfies (p) and its intersection is the set $g^{-1} g(x)$. Moreover, we can assume that $x \in E \in \mathcal{E}_{n}$ implies $x \in E^{\prime} \subset E$ for a certain $E^{\prime} \in \mathcal{E}_{n+1}$.

For $n \geqslant 1$ put $\mathscr{F}_{n}=\left\{f(E): E \in \mathcal{E}_{n}\right\}$ and observe that each $\mathscr{F}_{n}$ is a locally finite closed cover of $Y$ and $\left\{\operatorname{St}\left(y, \mathscr{F}_{n}\right)\right\}_{n \geq 1}$ satisfies (p) for $y \in Y$ (compare with [I, Lemma 2.2]).

Let $\mathscr{U}_{n}=\left\{U_{n}(y): y \in Y\right\}$, where $U_{n}(y)=Y \backslash \cup\left\{F \in \mathscr{F}_{n}: y \notin F\right\}$. It is easy to see that, for each $y \in Y$ and $n \geqslant 1, U_{n+1}(y) \subset U_{n}(y) \subset \operatorname{St}\left(y, \mathscr{F}_{n}\right)$ and $y \in U_{n}\left(y^{\prime}\right)$ implies $U_{n}(y) \subset U_{n}\left(y^{\prime}\right)$.

Take $U_{n}\left(y_{n}\right) \in \mathcal{Q}_{n}$ and assume that $y \in \bigcap_{n \geq 1} U_{n}\left(y_{n}\right)$. Then $y \in \bigcap_{n \geq 1} \operatorname{St}\left(y_{n}, \mathscr{F}_{n}\right)$ and consequently, $y_{n} \in \operatorname{St}\left(y, \mathscr{F}_{n}\right)$ for $n \geqslant 1$. Since $\left\{\operatorname{St}\left(y, \mathscr{F}_{n}\right)\right\}_{n \geq 1}$ satisfies (p), it follows that the sequence $\left\{y_{n}\right\}_{n \geq 1}$ has an accumulation point $y^{\prime} \in Y$.

We shall show that for each $n \geqslant 1$ there exists an $m \geqslant 1$ such that $U_{m}\left(y_{m}\right) \subset$ $\operatorname{St}\left(y^{\prime}, \mathscr{F}_{n}\right)$. This will imply, on one hand, that the sequence $\left\{U_{n}\left(y_{n}\right)\right\}_{n \geq 1}$ satisfies (p) - for $\left\{\operatorname{St}\left(y^{\prime}, \mathscr{F}_{n}\right)\right\}_{n \geq 1}$ satisfies (p) - and, on the other hand, that

$$
\begin{aligned}
\bigcap_{n \geqslant 1} U_{n}\left(y_{n}\right) & \subset \bigcap_{n \geqslant 1} \operatorname{St}\left(y^{\prime}, \mathscr{F}_{n}\right) \\
& =f\left(\bigcap_{n \geqslant 1} \operatorname{St}\left(f^{-1}\left(y^{\prime}\right), \mathcal{E}_{n}\right)\right)=f\left(g^{-1} g\left(f^{-1}\left(y^{\prime}\right)\right)\right) .
\end{aligned}
$$

Consequently, we shall obtain that $Y$ is a $p$-space and, according to the observation following our definition of $p$-spaces, $Y$ has a perfect mapping $h$ onto a metric space $T$ such that each fiber of $h$ is contained in a set $f\left(g^{-1} g\left(f^{-1}\left(y^{\prime}\right)\right)\right)$ for a certain $y^{\prime} \in Y$.

Thus, in order to finish the proof, we have to show that for each $n \geqslant 1, \operatorname{St}\left(y^{\prime}, \mathscr{F}_{n}\right)$ contains some $U_{m}\left(y_{m}\right)$. To this end, take $n \geqslant 1$ and an $m \geqslant n$ such that $y_{m} \in U_{n}\left(y^{\prime}\right)$. From the properties of the families $\left\{U_{n}(y): y \in Y\right\}$, we obtain $U_{m}\left(y_{m}\right) \subset U_{n}\left(y_{m}\right) \subset$ $U_{n}\left(y^{\prime}\right) \subset \operatorname{St}\left(y^{\prime}, \mathscr{F}_{n}\right)$. 
Observe that in the above proof we did not use the fact that paracompact $p$-spaces are preserved by perfect mappings.

2. Examples. We shall construct examples illustrating the results of the first section. Again, $X, Y, S$ and $f, g$ are as in the formulation of Theorem 1.1. The examples are modifications of the Alexandroff double circle [E, 3.1.26] which is a compact nonperfect space. The fact that in our constructions $X$ and $Y$ are not perfect spaces is, in view of [Ch2, Theorem 1.2.A], essential.

The first example shows that the assumption that the fibers of $f$ are countable in Corollaries 1.1 and 1.2 is essential and that one cannot claim that $y^{\prime}=y$ in Theorem 1.1 .

EXAMPLE 2.1. Let $X=A(C)$ be the Alexandroff double circle consisting of the topological circle $C_{0}$ and the set of isolated points $C_{1}$. The natural projection $g$ of $X$ onto $S=C_{0}$ is a two-to-one mapping and the mapping $f$ identifying $C_{0}$ to a point $y_{0}$ is a perfect mapping of $X$ onto $Y$ which is the Alexandroff compactification of the discrete space $C_{1}$. If $h$ maps $Y$ onto a metric space, then the fiber of $h$ containing $y_{0}$ is a $G_{\delta}$-set in $Y$ and consequently, has a countable completion in $Y$. Thus the fibers of $h$ are neither countable nor metrizable. If $y \in h^{-1} h\left(y_{0}\right)$ and $y \neq y_{0}$, then $f\left(g^{-1} g\left(f^{-1}(y)\right)\right)$ consists of two points and does not contain the fiber $h^{-1} h(y)$.

We shall need the following auxiliary example:

EXAMPLE 2.2. For each $m \geqslant 1$ there exists a compact space $A_{m}$ which has an $(m+1)$-to-one mapping onto a circle and is such that any mapping $h$ of $A_{m}$ onto a metric space has a fiber containing at least $m+1$ points.

The space $A_{m}$ is a natural modification of the Alexandroff double circle $A(C)$ which is exactly $A_{1}$. Namely, $A_{m}$ is the union of $m+1$ circles $C_{0} \cup C_{1} \cup \cdots \cup C_{m}$, where $C_{0} \subset A_{m}$ is a topological circle while $C_{1}, \ldots, C_{m}$ consist of isolated points of $A_{m}$ and are dense in $A_{m}$.

The topology of $A_{m}$ is such that the natural projection $p$ of $A_{m}$ onto $C_{0}$ is a continuous $\left((m+1)\right.$-to-one) mapping of $A_{m}$ onto the circle $C_{0}$. Moreover, $A_{m}$ has the property that any $G_{\delta}$-set in $A_{m}$, containing $C_{0}$, contains $p^{-1}(c)$ for all but a countable number of $c \in C_{0}$.

Assume that $h: A_{m} \rightarrow T$ is a mapping of $A_{m}$ onto a space $T$ with a metric $\rho$. Consider $r: A_{m} \rightarrow R$ defined by $r(y)=\rho(h(y), h p(y))$. Clearly $r$ is continuous and $r^{-1}(0)$ is a $G_{\delta}$-set in $A_{m}$ containing $C_{0}$. Let $c \in C_{0}$ be such that $p^{-1}(c) \subset r^{-1}(0)$. From the definition of $r$, it follows that $p^{-1}(c) \subset h^{-1} h(c)$, which shows that $h$ has a fiber containing at least $m+1$ points.

The next example shows that the cardinality of the fibers of $h$ in Corollary 1.3 cannot be decreased.

EXAMPLE 2.3. For each $k, n \geqslant 1$ there exists a compact space $X$ which has an $n$-to-one mapping onto a metric space $S$ and an at most $k$-to-one mapping $f$ onto the space $A_{m}$ from Example 2.2, where $m=k(n-1)$.

The space $X$ is the sum of $k$ copies of $A_{n-1}$. Clearly, $X$ has an $n$-to-one mapping $g$ onto the sum of $k$ circles $S$. On the other hand, the mapping $f$, identifying the corresponding points of $k$ circles corresponding to $C_{0}$ in each copy of $A_{n-1}$ in $X$, is a 
mapping of $X$ onto $Y=A_{m}$, where $m=k(n-1)$ and the fibers of $f$ are of cardinality not greater than $k$.

Observe that the above construction can be used in order to define $A_{m}$ from $A_{1}$. To obtain $A_{m}$ it is sufficient to put $n=2$ and $k=m$. The next example generalizes this construction.

EXAMPLE 2.4. For each compact metric space $M$, there exists a compact space $X$ which has a two-to-one mapping $g$ onto the metric space $M \times C$ and can be mapped by a mapping $f$, with nontrivial fibers homeomorphic to $M$, onto a space $A_{M}$ such that any mapping of $A_{M}$ onto a metric space has a fiber containing $M$ (in fact, it has a fiber containing the sum of $M$ and an isolated point).

Let $X=M \times A_{1}$. Clearly $g=i d \times p$, where $p$ is the projection of $A_{1}$ onto the circle $C_{0}$, is a two-to-one mapping of $X$ onto $M \times C_{0}$. On the other hand, the mapping $f$ which is the projection of $M \times C_{0} \subset X$ onto $C_{0}$ and the identity on $M \times C_{1}$, maps $X$ onto a space which we denote, according to the notation of Example 2.2, by $A_{M}$. The proof of the fact that any mapping of $A_{M}$ onto a metric space has a fiber containing $M$ (plus an isolated point) is the same as in Example 2.2.

If $M$ is the convergent sequence, then we obtain an example illustrating the part of Corollary 1.2 concerning finite-to-one mappings.

EXAMPLE 2.5. There exists a compact space $X$ which has a two-to-one mapping onto a metric space and can be mapped by a countable-to-one mapping $f$ onto a space $Y$ such that any mapping of $Y$ onto a metric space has an infinite fiber.

The above constructions can be generalized still further:

If $e: P \rightarrow M$ is a perfect mapping of a paracompact $p$-space $P$ onto a metric space $M$, then one can define a paracompact $p$-space $A_{e}$, by identifying in $P \times A_{1}$ each set of the form $e^{-1}(m) \times\{c\}$, where $m \in M$ and $c \in C_{0}$, to a point. Thus the quotient space $A_{e}=\left(M \times C_{0}\right) \cup\left(P \times C_{1}\right)$ has a natural projection onto $M \times C_{0}$ which is perfect and has the fibers corresponding to the fibers of $e$. Moreover, any mapping of $A_{e}$ onto a metric space has to have fibers corresponding to the fibers of $e$.

If $e_{0}: P \rightarrow M$ and $f_{0}: M \rightarrow M^{\prime}$ are perfect mappings and $M, M^{\prime}$ are metric spaces, then $X=A_{e_{0}}$ has a perfect mapping $f$ generated by $f_{0}$ onto $Y=A_{e}$, where $e=f_{0} e_{0}$. If $h$ is a mapping of $Y$ onto a metric space, then for each $m \in M^{\prime}$, there exists a $c \in C_{0}$ such that $y=(m, c) \in M^{\prime} \times C_{0} \subset Y$ satisfies $h^{-1} h(y) \supset f\left(g^{-1} g\left(f^{-1}(y)\right)\right)$, where $g$ is the natural projection of $X$ onto $M \times C_{0}$.

Observe that for $M=\{1, \ldots, k\}, P=M \times\{1, \ldots, n-1\}, e_{0}$ being the projection of $P$ onto $M$ and $f_{0}$ identifying $M$ to a point, the above construction becomes our Example 2.3.

3. Perfect images of $p$-spaces. It is known that perfect images of $p$-spaces satisfying certain covering properties such as metacompactness, subparacompactness or, more generally, submetacompactness $(=\theta$-refinability) are $p$-spaces (see $[\mathbf{B}$, Table 1]). We shall use an example from [DGN, D] and a method from [W] in order to construct an example of a perfect mapping $f$ of a $p$-space $X$ with a $\sigma$-locally countable and $\sigma$-disjoint base onto a space $Y$ which is not a $p$-space. This shows that the covering properties listed above cannot be replaced by the property of being metalindelöf nor even screenable and $\sigma$-paralindelöf space [FR]. 
First we need the example from [DGN, Example 3.3 and D].

EXAMPLE 3.1. There exists a Čech complete space $Z$ with a $\sigma$-locally countable and $\sigma$-disjoint base containing a discrete closed subset $F$ which is not a $G_{\boldsymbol{\delta}}$-set in $Z$.

EXAMPLE 3.2. There exists a perfect mapping $f$ of a $p$-space $X$ with a $\sigma$-locally countable and $\sigma$-disjoint base onto a space $Y$ which is not a $p$-space.

Let $Z$ be the space from Example 3.1 and $M=\{0\} \cup\left\{\frac{1}{n}: n \geqslant 1\right\}$ the convergent sequence. Put $X=Z \times M \backslash((Z \backslash F) \times\{0\})$. Clearly, $X$ has a $\sigma$-locally countable and $\sigma$-disjoint base. To see that $X$ is a $p$-space, assume that $\left\{\mathcal{U}_{k}\right\}_{k>1}$ is a decreasing sequence of open covers of $Z$ such that $U_{k} \in \mathcal{U}_{k}$ for $k \geqslant 1$ implies that $\left\{U_{k}\right\}_{k>1}$ satisfies (p). Moreover, since $F$ is closed and discrete in $Z$, we can assume that $z \in F$ and $z \in \bigcap_{k>1} U_{k}$ implies that $\left\{U_{k}\right\}_{k>1}$ forms a base of neighbourhoods of $z$ in $Z$.

Define

$$
\begin{aligned}
\mathscr{V}_{k}= & \left\{X \cap\left(U \times\left(\{0\} \cup\left\{\frac{1}{n}: n \geqslant k\right\}\right)\right): U \in \mathscr{Q}_{k}\right\} \\
\cup & \bigcup_{n \geq 1}\left\{U \times\left\{\frac{1}{n}\right\}: U \in \mathscr{Q}_{k}\right\} .
\end{aligned}
$$

Consider a sequence $\left\{V_{k}\right\}_{k>1}$ such that $V_{k} \in \mathscr{V}_{k}$ and suppose that $x \in \bigcap_{k>1} V_{k}$. If $x=\left(z, \frac{1}{n}\right)$, then $V_{k}=U_{k} \times\left\{\frac{1}{n}\right\}$ for $k>n$. If $x=(z, 0)$, then $z \in F, V_{k}=X \cap\left(U_{k}\right.$ $\left.\times\left(\{0\} \cup\left\{\frac{1}{n}: n \geqslant k\right\}\right)\right)$ and $z \in \bigcap_{k \geq 1} U_{k}$, which shows that $\left\{V_{k}\right\}_{k>1}$ forms a base of neighbourhoods of $x$ in $X$. Thus, in both cases, $\left\{V_{k}\right\}_{k>1}$ satisfies (p) and consequently, $X$ is a $p$-space.

The quotient mapping $f$ identifying each set of the form $\{z\} \times M$, where $z \in F$, to a point is perfect. It remains to show that the quotient space $Y$ is not a $p$-space.

Assume that $\left\{W_{k}\right\}_{k>1}$ is a sequence of open covers of $Y$. For each $z \in F$ and $k \geqslant 1$ find an open in $Z$ set $G_{k}(z)$ and an element $W_{k}(z)$ of $W_{k}$ such that $\{z\} \times M \subset X \cap\left(G_{k}(z) \times M\right) \subset f^{-1}\left(W_{k}(z)\right)$. Since $F$ is not a $G_{\delta}$-set in $Z$, it follows that there exists a $z \in Z \backslash F$ and a sequence $\left\{z_{k}\right\}_{k>1}$ of elements of $F$ such that $z \in \cap_{k>1} G_{k}\left(z_{k}\right)$. Then $\{z\} \times\left\{\frac{1}{n}: n \geqslant 1\right\} \subset \bigcap_{k>1} W_{k}\left(z_{k}\right)$, which shows that $\left\{W_{k}\left(z_{k}\right)\right\}_{k>1}$ does not satisfy (p).

A technique for constructing examples such as Example 3.2 is implicit in [W] and the verification of Example 3.2. The following remark has a similar proof:

REMARK 3.3. Suppose $Z$ is a $p$-space containing a closed discrete subset $F$ where $F$ is not a $G_{\delta}$-set but every element of $F$ has a countable local base. If $X=Z \times$ $M \backslash((Z \backslash F) \times\{0\})$ then $X$ is a $p$-space and the quotient mapping $f$ obtained by identifying each set $\{z\} \times M$, where $z \in F$, to a point is a perfect mapping onto a quotient space $Y$ which is not a $p$-space.

The following problem seems to be natural in view of the above remark:

Problem 3.4. Let $f: X \rightarrow Y$ be a perfect mapping of a $p$-space $X$ onto a perfect space $Y$. Is $Y$ a $p$-space?

\section{REFERENCES}

[A] A. V. Arhangel'skii, On a class of spaces containing all metric spaces and all locally bicompact spaces, Amer. Math. Soc. Transl. (2) 92 (1970), 1-39.

[B] D. K. Burke, Closed mappings, Surveys in General Topology, Academic Press, New York, 1980, pp. $1-32$.

[Ch1] J. Chaber, Mappings onto metric spaces, Topology Appl. (to appear). 
[Ch2] __ Closed mappings onto metric spaces, Topology Appl. (to appear).

[D] S. W. Davis, $A$ nondevelopable Čech complete space with a point-countable base, Proc. Amer. Math. Soc. 78 (1980), 139-142.

[DGN] S. W. Davis, G. Gruenhage and P. J: Nyikos, $G_{\delta^{-}}$sets in symmetrizable and related spaces, General Topology and Appl. 9 (1978), 253-261.

[E] R. Engelking, General topology, PWN, Warsaw, 1977.

[F] V. V. Filippov, On the perfect image of a paracompact p-space, Soviet Math. Dokl. 8 (1967), $1151-1153$.

[FR] W. G. Fleissner and G. M. Reed, Paralindelöf spaces and spaces with a $\sigma$-locally countable base, Topology Proc. 2 (1977), 89-110.

[I] T. Ishii, On closed mappings and M-spaces. I, II, Proc. Japan Acad. 43 (1967), 752-761.

[P] C. M. Pareek, Characterizations of p-spaces, Canad. Math. Bull. 14 (1971), 459-460.

[W] J. M. Worrell, Jr., A perfect mapping not preserving the p-space property (preprint).

Institute of Mathematics, University of Warsaw, 00-901 Warsaw, Pkin, Poland 